\title{
Sonographic Comparison of Portal Vein Diameter in Cirrhotic and Non-Cirrhotic Patients
}

\author{
Kiran tahir ${ }^{1}$, Dr. Raham bacha ${ }^{1}$, Dr.Saira Bilal ${ }^{1}$, Sheza Waseem ${ }^{1}$, Ali Raza ${ }^{1}$, Iqra ${ }^{1}$, Dr. Saifullah ${ }^{1}$, Dr. Syed \\ Hasanain Shah ${ }^{2}$. \\ ${ }^{1}$ University Institute of Radiological Sciences and Medical Imaging Technologies, Faculty of Allied Health \\ Sciences, University of Lahore, Lahore, Pakistan. \\ ${ }^{2}$ The University of Lahore. \\ Corresponding Author: Kiran Tahir \\ Corresponding Email: kirantahir1310@gmail.com
}

\begin{abstract}
:
Background: Liver cirrhosis has become one of the major causes of morbidity and mortality. The burden of liver cirrhosis is growing in both the West and the East. In Pakistan death rate of liver cirrhosis is conspicuous because of chronic hepatitis (hepatitis B, C) and hepatocellular carcinoma. It is reported by World Health Organization that Pakistan occupies secondary place in spread of hepatitis C.
\end{abstract}

Objective: To compare the portal vein diameter in cirrhotic and non-cirrhotic patients through ultrasound.

Methodology: Ultrasound machine Toshiba Xario, Mindray dp 10 and G logic p5 with a curvilinear transducer of frequency 3.5 MHz was used. The study was conducted at, Hussain Diagnostic Ultrasound Centre Jampur, District Rajanpur. Data of 100 patients was collected through Cross-Sectional Analytical study. Statistical software for social sciences (SPSS version 22.0) is used for the analysis of data.

Results: One hundred patients participated in this study. Among them, the minimum age was 30 and the maximum age was 70. Ratio of male patients was more than female patients, due to fact of more alcohol consumption in males. Out of 100 patients, 50 patients had cirrhosis and 50 were non cirrhotic. Liver cirrhosis patients came the severe symptoms like weakness, lethargy, hematemesis and melena. Non cirrhotic patients came with epigastric pain, nausea and vomiting. Mean of Portal vein diameter in non-cirrhotic patients was $10.5 \mathrm{~mm}$. Mean of Portal vein diameter in live cirrhosis patients was $14.8 \mathrm{~mm}$. A statistical significance difference was found between the two means of portal vein diameter of two groups (cirrhotic and non-cirrhotic) as the p-value 0.000 less than 0.05 .

Conclusion: Liver cirrhosis is one of the major issues of health and a big reason for increasing mortality rate all over the world. The most common etiology of liver cirrhosis is alcohol. Patients come with liver cirrhosis having severe symptoms like weakness, lethargy, hematemesis and melena. Non-cirrhotic patients come with mild symptoms like epigastric pain, nausea and vomiting, having normal portal vein diameter. Mean portal vein diameter in cirrhotic patients $(14.8 \mathrm{~mm})$ was greater than non-cirrhotic patients $(10.5 \mathrm{~mm})$.

Key words: Liver cirrhosis, Non-alcoholic steatohepatitis (NASH), Non- alcoholic fatty liver disease (NAFLD), alcoholic liver disease (ALD).

DOI: $10.7176 / \mathrm{JHMN} / 76-12$

Publication date:June 30th 2020

\section{INTRODUCTION:}

Liver cirrhosis is one of the major issues of health and a big reason for increasing mortality rate all over the world. According to Global Burden of Disease (GBD) over one million people died due to cirrhosis in 2010 worldwide. ${ }^{1}$ According to autopsy studies worldwide occurrence of cirrhosis, measured from $4.5 \%$ to $9.5 \%$ in 
ubiquitous community. In 2001, the evaluated universal death rate from cirrhosis was 771,000 population, grading 14th and 10th as the foremost etiology of demise in the domain and in advanced nations, appropriately. Cirrhosis will be a $12^{\text {th }}$ major issue of demise in 2020. Universally, usual etiologies of cirrhosis are viral hepatitis, alcohol, non-alcoholic steatohepatitis (NASH). Preponderance of cirrhosis is possibly to be underrated as about a 3 rd of the sufferers remain undetermined. ${ }^{2}$

Cirrhosis is the main cause of illness and fatality in the United States. It was specified by Centers for Disease Control and Prevention that cirrhosis was the $12^{\text {th }}$ key factor of demise in the United States in 2013, reporting for over 36,000 deaths. Non- alcoholic fatty liver disease (NAFLD) is the superiorly significant source of cirrhosis. Alcohol remains to be one of the large donors to hepatic disorder in the United States. It is clear that alcoholic liver disease (ALD) is the greatest etiology of cirrhosis in whites. According to recent data from the National Institute on Alcohol Abuse and Alcoholism demonstrated that whites are mostly expire from alcoholic liver cirrhosis as compared to blacks. ${ }^{3}$ In Europe, about $0.1 \%$ of Hungarian males will die of cirrhosis every year compared with $0.001 \%$ of Greek females. ${ }^{4}$

In Pakistan death rate of liver cirrhosis is conspicuous because of chronic hepatitis (hepatitis B, C) and hepatocellular carcinoma. It is reported by World Health Organization that Pakistan occupies secondary place in spread of hepatitis C. There are about 1,000,000 persistent patients of hepatitis B and 1,700,000 persistent patients of hepatitis $\mathrm{C}$ in Sind province of Pakistan. One main reason of death rate across Pakistani population is cirrhosis, typically the most frequent complexity of cirrhosis with portal hypertension are esophageal varices. Ascites were present in 59\% of patients in Pakistan. Spontaneous bacterial peritonitis (SBP) contributes almost $24 \%$ of in death rate in Pakistan, mainly in Sindh. Universally $6^{\text {th }}$ frequently occurring cancer is hepatic cancer; which affects every $12^{\text {th }}$ male and every $4^{\text {th }}$ female per 100,000 males and females respectively, in Pakistan. HCC broadly (96\%) experienced in post cirrhotic liver in persistent hepatitis C diseased patients. ${ }^{5}$

Liver carries out a series of activities that assist metabolism, immunity, digestion, detoxification, vitamin storage among other activities. ${ }^{6}$ Its main tasks are to detoxify various metabolites, synthesize proteins, and generate enzymes essential for digestion. The liver also has an important role in metabolism, management of red blood cells (RBCs) and glucose composition and retention. ${ }^{7}$ The liver gathers its blood delivery from two resources: $80 \%$ is supplied by the portal vein, which drains the spleen and intestines; the remaining $20 \%$, the oxygenated blood, is supplied by the hepatic artery. ${ }^{8}$

Cirrhosis is a significant deteriorative disease in which interchange of normal liver tissue with fibrous tissue takes place that results in distortion of liver anatomy and performance. In patients with persistent hepatic disease, portal hypertension and cirrhosis are obvious. Current progress in the interpretation of the usual report and morbid physiology of cirrhosis, and in cure of its problems, leading to enhanced administration, living standards and survival rate of cirrhotic patients. Now a days, the single remedial choice for a preferred panel of sufferers is liver transplantation, but progression of pharmacological drugs have sopped the development of decompensated cirrhosis or while converse cirrhosis are presently being augmented. ${ }^{9}$

\section{RESULTS}

One hundred patients participated in this study. Among them, the minimum age was 30 and the maximum age was 70. Out of 100 patients, 50 patients had cirrhosis and 50 were non-cirrhotic. Patients came with liver cirrhosis had severe symptoms like weakness, lethargy, hematemesis and melena. Non-cirrhotic patients came with mild symptoms like of epigastric pain, nausea and vomiting. Mean of Portal vein diameter in non-cirrhotic patients was $10.5 \mathrm{~mm}$ with standard deviation of 1.613 . Mean of Portal vein diameter in live cirrhosis patients was $14.8 \mathrm{~mm}$ with standard deviation of 1.244 , according to table 1. A statistical significance difference was found between the two means of portal vein diameter of two groups (cirrhotic and non-cirrhotic) as the p-value 0.000 less than 0.05 , according to table 2 . A detail description is given below 


\begin{tabular}{lc|c|c|c} 
& $\mathrm{N}$ & Mean $(\mathrm{mm})$ & Std. Deviation & Std. Error Mean \\
\hline Non cirrhotic & 50.000 & 10.550 & 1.613 & 0.228 \\
cirrhosis & 50.000 & 14.890 & 1.244 & 0.176 \\
\hline
\end{tabular}

Table 1: Mean of portal vein diameter (PVD) in cirrhosis and non-cirrhotic patients.

\begin{tabular}{l|l|l|l|l|r} 
& Mean & Std. Error & $t$ & df & \multicolumn{1}{l}{ Sig. (2-tailed) } \\
\hline Equal variances assumed & -4.340 & .288 & -15.066 & 98.000 & .000 \\
\hline $\begin{array}{l}\text { Equal variances not } \\
\text { assumed }\end{array}$ & -4.340 & .288 & -15.066 & 92.057 & .000 \\
\hline
\end{tabular}

Table 2: Independent Samples Test

\section{DISCUSSION}

One hundred patients participated in our study. Among them, the minimum age was 30 and the maximum age was 70. Out of 100 patients, 50 patients had cirrhosis and 50 were control. In current study, there were more male patients than female patients. A .study .done .by .Ndububa .et .al in 2010; in .South-Western .Nigeria .on .145 .patients .on .the .contribution .of .alcohol .to development .of .CLD had .more .males .(102) .than .females .(43)10. .A study of 108 CLD patients by Kamran et al on correlation between sonographic PV diameter and flow velocity in cirrhotic patients also had more males (66) than females (42).

In .our .study, .the .most .common .etiology .of .cirrhosis .was .alcohol. .General .symptoms .found .in .our .cirrhotic .patients .were weakness, hematemesis and melena. Non cirrhotic patients came with the symptoms of epigastric pain, vomiting, weakness and nausea.

Maaji .et .al in 2016 also had .male .predominance in .his .study .on .sonographic .findings .of .CLD .patients in Sokoto. .These .findings .concorded .with .this .study .confirming .male .predominance in .CLD in .our environment. .The .male .dominance .in .this .study .was .possibly .due to .high .alcohol .intake .and increased risk .of .hepatitis .B .infection .which .increases .the .risk .of .the .disease11. In our study male dominance was also seen.

In current study, mean of portal vein diameter in patients with non-cirrhotic came 10.5 with standard deviation of 1.613. Mean of portal vein diameter in patients with live cirrhosis came 14.8 with standard deviation of 1.244 , according to table 15 and figure 4 . Similar findings were found by other researchers. A .study .done .by Lopamudra et .al.in 2011 on .82 .CLD .patients .found .a .higher .value .of .mean .PV .diameter .of .13.99 . . .1.12 mm12. Nizar .et .al. .also .reported .an .increased .PV .diameter .of .up .to .17 .mm13. .In .another .study Rina Mohanty et al 2017 on .107 .CLD .patients .also .found .an .increase .in .diameter .of .the .extrahepatic .PV .which .was .>13 mm14. .Similar studies .done .also showed .increase .in .diameter .of the .main .PV. Hawaz Y in 2009, studied the .mean .diameter .of .the .main .PV .in .CLD .patients .was .higher .(18.68...2.59 .mm) than .that .of .the control .(10.87. $\pm .0 .81 \mathrm{~mm})$. .The .variation .in the .values .of .the .main .PV .diameter .in .the .various .studies may .be .due to the difference in sample .size, ethnic .and .geographical differences .between .the populations studied15. .Hawaz .et .al. .reported .an .increase .in .diameter .of.PV .with .increase .in .age .in .normal individuals which .is .consistent .with .the .finding .of .Anakwue .et .al done in 200915,17. .Similar .findings were .also .found by .many .researchers i.e, Shankar RG et al in 2011 and by Subramanyam BR in 198918,19.

In current study, a statistical significance difference found between the two means of portal vein diameter of two groups (cirrhotic and non-cirrhotic) as the p-value 0.000 less than 0.05 . Aminu Umar Usman et al. .in .his .study 
on .191.CLD .patients .and .247 .controls .reported .no .difference .in .PV .diameter .values .between .male .and females .in .both .groups16. . .However, .Hawaz .et .al. .and .Anakwue .et .al. .found .correlation .between .PV diameter .with .sex .in .CLD .patients, .but .they .found .no .correlation .between .PV .diameter .and .sex .in .normal subjects. .This .study .found .no .significant .difference .PV .diameter .in .male .and .females .in .CLD .patients (P . .0.005). .However, there .was .a .statistical difference .in .the .control .group . $(\mathrm{P} .<.0 .05)$.with .values .slightly higher .in .females than .males19,20.

A .study .by . Shankar RG et al .on .50 .CLD .patients .and .50 .controls. This .study .found a .significant difference between .right .and .left .PV .diameter .in .male .CLD .patients .and .control .group . $(\mathrm{P} .<.0 .005)$. .No difference was, however, found .in .female .CLD .patients .(P .> .0.005)69. Bolondi L et al .found .a .mean .portal .vein

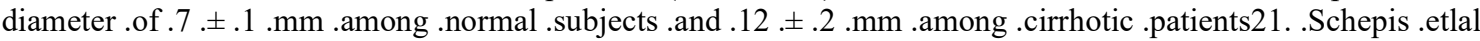
10 .found .a .portal .vein .diameter .of .13.82. $\pm .2 .1 \mathrm{~mm}$. .Prihatini .et .al 11 .concluded .in .their .study .that portal vein .size $.1 .2-\mathrm{cm} 22$

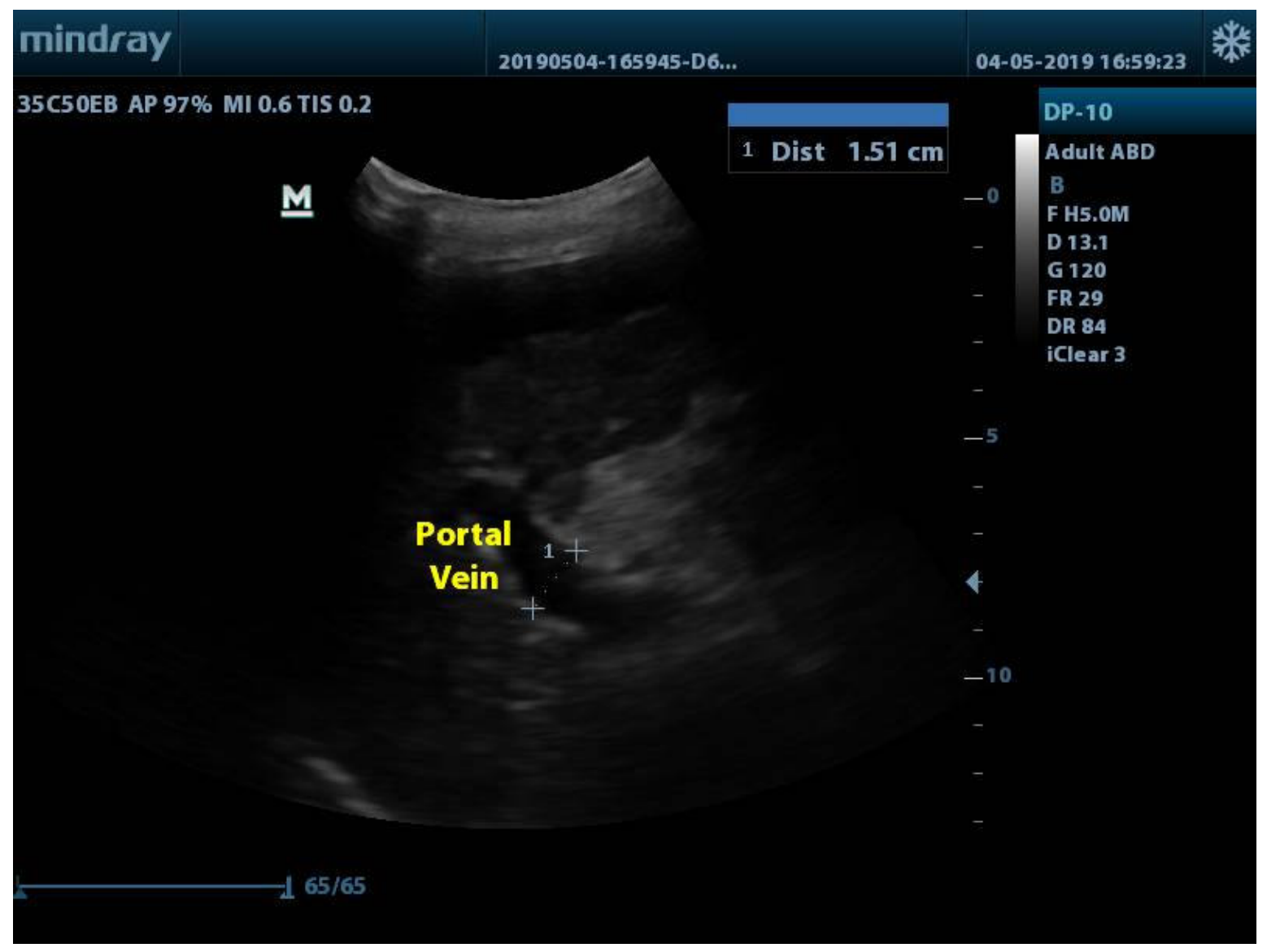

Figure 1: TA gray scale image showing, portal vein diameter of $1.51 \mathrm{~cm}$. 


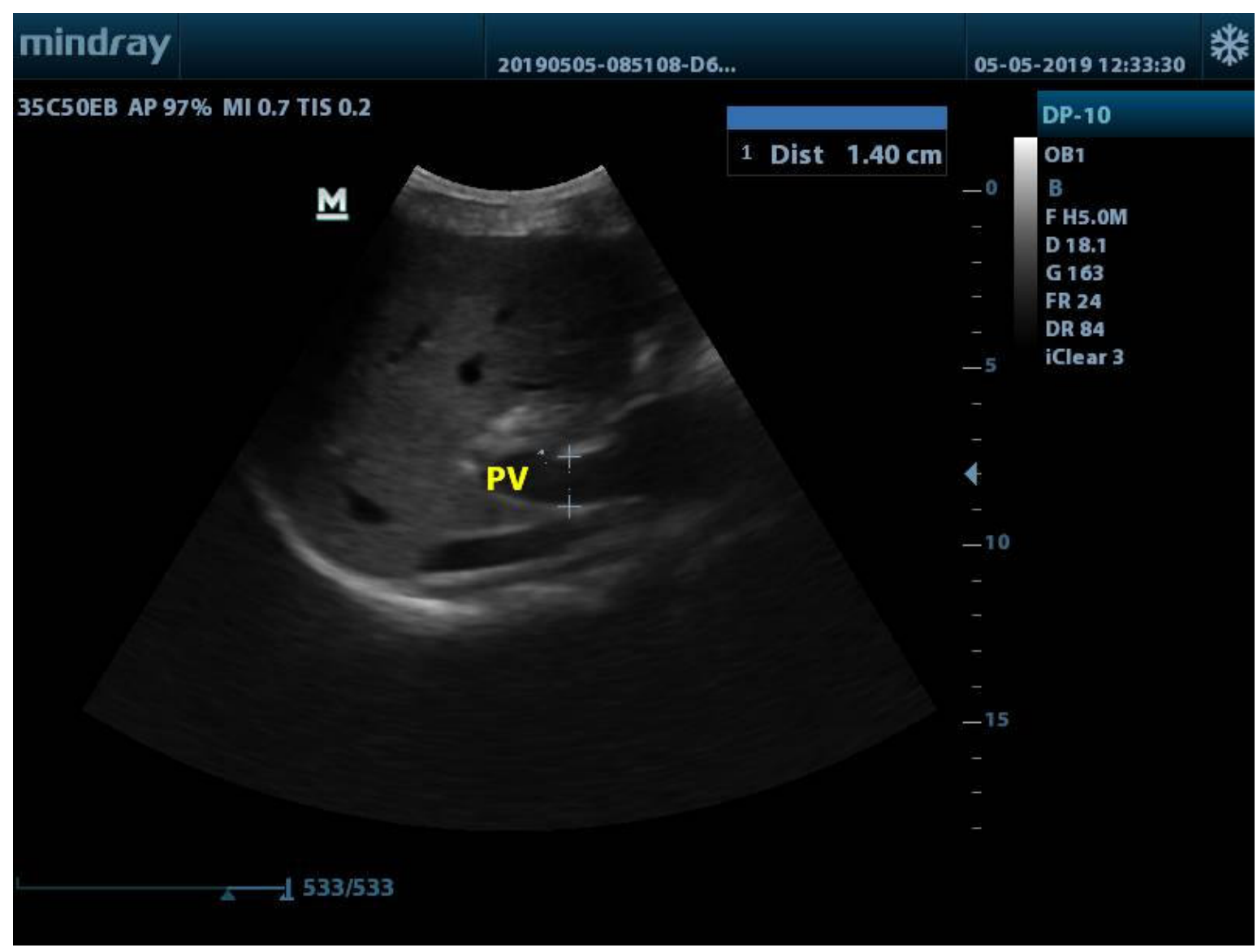

Figure 2: TA gray scale image showing, portal vein diameter of $1.4 \mathrm{~cm}$.

\section{REFERENCES}

1. Wong MC and Huang J. The growing burden of liver cirrhosis: implications for preventive measures. Springer, 2018.

2. Sarin S and Maiwall R. Global burden of liver disease: a true burden on health sciences and economies. World Gastroenterol Organ 2012; 17: 35-40.

3. Setiawan VW, Stram DO, Porcel J, et al. Prevalence of chronic liver disease and cirrhosis by underlying cause in understudied ethnic groups: the multiethnic cohort. Hepatology 2016; 64: 1969-1977.

4. Blachier M, Leleu H, Peck-Radosavljevic M, et al. The burden of liver disease in Europe: a review of available epidemiological data. Journal of hepatology 2013; 58: 593-608.

5. Memon MS and Zaki M. Burden of chronic liver disease and liver transplantation in Sindh. JLUMHS 2013; 12: 01 .

6. Kalra A and Tuma F. Physiology, Liver. StatPearls [Internet]. StatPearls Publishing, 2018.

7. Lala V and Minter DA. Liver Function Tests. StatPearls [Internet]. StatPearls Publishing, 2018.

8. Sibulesky L. Normal liver anatomy. Clinical liver disease 2013; 2: S1.

9. Schuppan D and Afdhal NH. Liver cirrhosis. The Lancet 2008; 371: 838-851. 
10. Ndububa DA, Ojo OS, Adetiloye VA, Aladegbaiye AO, Adebayo RA, Adekanle O. The contribution of alcohol to chronic liver disease in patients from South-west Nigeria. Niger J Clin Pract 2010;13:360-4

11. Maàji SM, Yakubu A, Odunko DD. Pattern of abnormal ultrasonographic findings in patients with clinical suspicion of chronic liver disease in Sokoto and its environs. Asian Pacific Journal of Tropical Disease. 2013 Apr 1;3(3):202-6.

12. Ivanova II. Liver cirrhosis: New concepts. Scripta Scientifica Medica 2016; 48: 9-16.

13. Nizar A. Role of ultrasonography in portal hypertension. Saudi Journal of Gastroenterology 2006;12:111-7.

14. Rina Mohanty, Namita Mohapatra et al.2017, correlation of portal vein diameter and splenic size with oesophageal varices in cirrhosis of liver, j.Evolution Med . Dent. Sci.6(82), 5746-5749

15. Hawaz Y, Admassie D, Kebede T. Ultrasound Assessment of Normal Portal Vein Diameter in Ethiopians Done at Tikur Anbessa Specialized Hospital. East Cent Afr J Surg. 2012; 17 (1):90-93.

16. Aminu Umar Usman1 PI, Ahmed Ahidjo3, Abdurrahman Tahir3, Sulaiman Tanimu Sa'ad1, Zainab Mustapha3, Nasiru Tahir1, Sani Garko1. Ultrasound Determination of Portal Vein Diameter in Adult Patients with Chronic Liver Disease in North-Eastern Nigeria. (C) Sub-Saharan African Journal of Medicine 2015; 2 Page : 57-63. DOI: 10.4103/2384-5147.157419

17. Anakwue AC, Anakwue RC, Ugwu AC, et al. Sonographic evaluation of normal portal Vein diameter in Nigerians. European Journal of scientific research. 2009; 36 (1): 114-17

18. Shankar RG, Shailaja S, Srinath MG, Roopa K. Estimation of portal vein diameter in corelation with age, sex and height of an individual. Anat Karnataka 2011;5:13-6.

19. Subramanyam BR, Balthazar EJ, Madamba MR, Raghavendra BN, Horii SC, Lefleur RS. Sonography of portosystemic venous collaterals in portal hypertension. Radiology 1983;146:161-6.

20. Hawaz Y, Admassie D, Kebede T. Ultrasound assessment of normal portal vein diameter in Ethopians at Tikur Anbessa Specialized Hospital. East Cent Afr J Surg 2012;17:90-3.

21. Bolondi L, Mazziotti A, Arienti V et al. Ultrasonographic study of portal venous system in portal hypertension and after portosystemic shunt operations. Surgery 1984; 95: 261-9.

22. Prihartini J, Lesmana LA, Manan C, Gani RA. Detection of esophageal varices in liver cirrhosis using non-invasive parameters. portal. 2005 Jul 20;37(3) 